“ (C) 2018 IEEE. Personal use of this material is permitted. Permission from IEEE must be obtained for all other uses, in any current or future media, including

reprinting/republishing this material for advertising or promotional purposes, creating new collective works, for resale or redistribution to servers or lists, or reuse of any copyrighted component of this work in other works." 


\section{A 28-GHz, Multi-Layered, Circularly Polarized, Electrically Small, Huygens Source Antenna}

\author{
Ming-Chun Tang, and Ting Shi \\ College of Communication Engineering, Chongqing \\ University, Chongqing, 400044, China \\ E-mail: tangmingchun@cqu.edu.cn, tingshi@cqu.edu.cn
}

\author{
Richard W. Ziolkowski ${ }^{1,2}$ \\ ${ }^{1}$ University of Technology Sydney, Global Big Data \\ Technologies Centre, Ultimo NSW 2007, Australia, and the \\ ${ }^{2}$ Department of Electrical and Computer Engineering, \\ University of Arizona, Tucson, AZ 85721, USA \\ E-mail: Richard.Ziolkowski@uts.edu.au
}

\begin{abstract}
A 28 GHz, multi-layered, circularly- polarized (CP), Huygens source electrically small antenna (ESA) is presented. The CP radiation is realized by integrating two orthogonal linearly polarized Huygens source EASs into one with an asymmetrical arc strip feed providing the necessary $90^{\circ}$ phase difference. Finally, the CP Huygens source ESA exhibits the properties: $k a=0.942 ; 1.41 \% \mathrm{FBW}_{-10 \mathrm{~dB}}$ with a $0.47 \% 3-\mathrm{dB}$ axial ratio (AR) fractional bandwidth; and peak realized gain, FTBR, and radiation efficiency values are $2.03 \mathrm{dBi}, 26.72 \mathrm{~dB}$, and $73.4 \%$, respectively. To confirm its efficacy for on-body applications, the specific absorption rate (SAR) values of the Huygens source ESA is evaluated and found to be very low.
\end{abstract}

Keywords-broadside radiation, electrically small antenna, circularly polarized, huygens source antenna, planar antenna

\section{INTRODUCTION}

With the rapid development of wireless communication, the frequency spectrum below $6 \mathrm{GHz}$ appears more crowded, and the demands of higher data rates are more intense. Therefore, the evolution of wireless access technologies is about to reach its fifth generation (5G), probably exploiting millimeter Wave (mmWave), where communication at 28 $\mathrm{GHz}, 38 \mathrm{GHz}$ center frequencies is foreseen as the most promising choice[1]. The $5 \mathrm{G}$ applications should call for antennas with easy to form an array and easy to integrate. Therefore, single antenna with the high directional radiation performances, physically smaller size and planar architecture would be enthusiastically desired. As generalizing in [2], there are a variety of approaches to realize the higher directivity of the electric small antennas (ESAs). Particularly, Huygens source antennas, due to their inborn beautiful quality in the directional radiation performances, are received widespread attention. As an alternative, we have recently achieved several single-feed, electrically small, near-field resonant parasitic (NFRP) [3] Huygens source antennas, e.g., coplanar stripline (CPS)-fed, end-fire radiating [4]; low profile, broadside radiating [5], [6], pattern-reconfigurability [7] and broader bandwidth non-Foster [8] versions.

In this paper, as the further reach of the current work in [38], a 28-GHz, multi-layers, planar Huygens source ESA with high direction and easy to integration is explored to cater the demand of the $5 \mathrm{G}$ communications. Due to the feature of relative insensitivity to transmitter and receiver orientations

This work was supported in part by the National Natural Science Foundation of China contract number 61471072 , in part by the Fundamental Research Funds for the Central Universities contract number 106112015CDJZR165510, in part by China Postdoctoral Science Foundation contract number 2016M590860, in part by Funding of the Young Backbone Teachers in Colleges and Universities of Chongqing contract number 0307001104102, and in part by the Australian Research Council grant number DP160102219. for the circularly polarized antennas, a circularly polarized version will be introduced. Furthermore, considering in some certain application scenes, such as the wearable equipment and the handset, there has an unavoidable close contact between the human body and the antenna. The biological implications of the antenna on the user have been investigated though the specific absorption rate (SAR) values.

\section{Design OF HuYGENS SOURCE CP ESA}

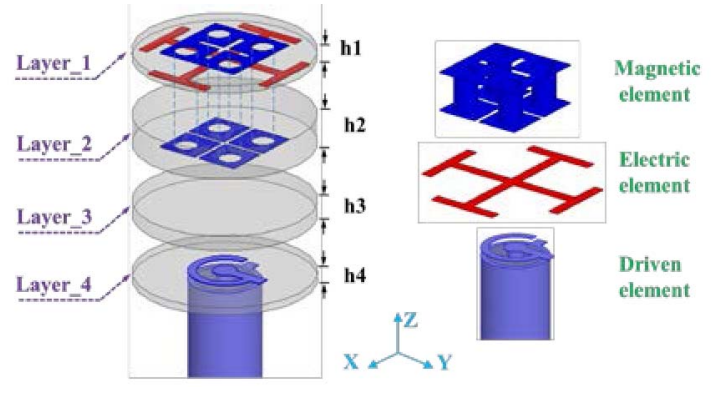

(a)
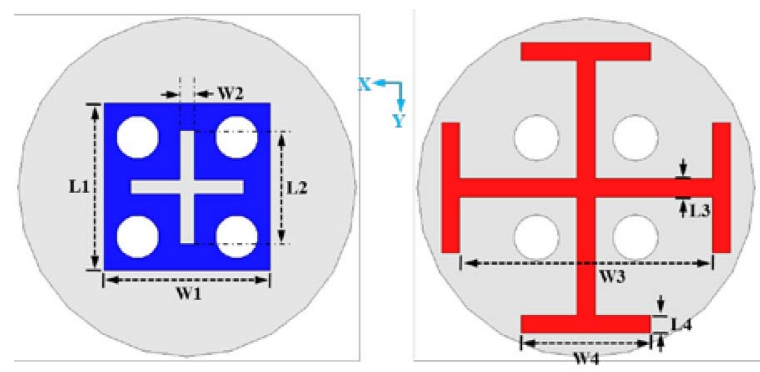

(b)
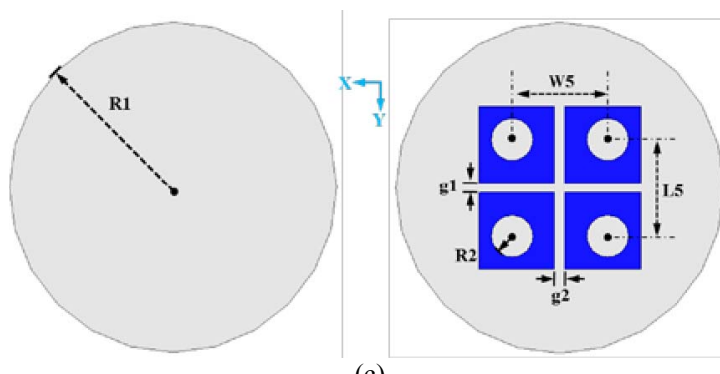

(c) 

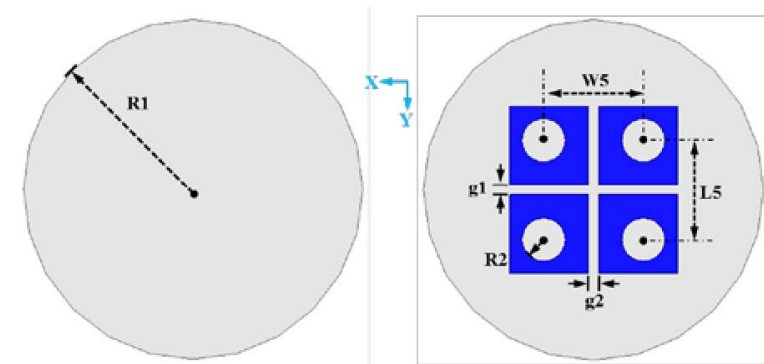

(d)

Fig.1 Geometry of the CP Huygens source ESA. (a) 3-D isometric view; (b) upper and lower surface of Layer 1; (c) upper and lower surface of Layer_2; and (d) upper and lower surface of Layer_4.

As shown in Fig. 1, the CP Huygens source ESA consists of four-layer Rogers Duroid TM 5880 substrates and including three parts, the magnetic element, the electric element and the driven element. Taking advantage of the successful near-field resonant parasitic (NFRP) approach [2-8], both the magnetic and electric elements are placed in the near field of the driven element and are excited by it. The electric NFRP element consists of two "I" elements oriented orthogonal to each other, and lies on the lower surface of the Later_1, as shown in Fig. 1(b). The magnetic NFRP element consists of a square patch with cross slot, a square patch with cross gap and four vertical metallic holes. The four metallic holes are used to connect the two square patches, which are on the upper side of Layer 1 and the lower side of Layer_2, respectively. For the square patch with cross slot on the upper side of Layer_1, the cross slot is determined to improve the purity of the resulting $\mathrm{CP}$ radiation, and does help to adjust the capacitive coupling strength between the magnetic NFRP element and the driven element. For the square patch with cross gap on the lower side of Layer_2, the cross gap is capacitive gap of capacitively loaded loop acting as the magnetic element. The driven element is constituted of a $50 \mathrm{ohm}$ coaxial line and two parts of mutually orthogonal feed lines, which are located on the upper and the lower surface of the Layer_4, respectively. The two orthogonal feed lines are connected to the inner and outer conductor of the $50 \Omega$ coaxial line, respectively. Especially, the feed line, which is located on the upper surface of Layer 4, has an additional arc-shaped strip. The arc-sharped strip is necessary in making 90 phase difference and does great help with the CP characteristic design. The electrical size of the Huygens source ESA is $0.28 \lambda_{\mathrm{L}} \times 0.28 \lambda_{\mathrm{L}} \times 0.107 \lambda_{\mathrm{L}}$ (where $\lambda_{\mathrm{L}}$ indicates the free space wavelength corresponding to $28 \mathrm{GHz}$ ). The antenna was simulated in HFSS and certified in CST. The final optimized parameters are given in Table I.

TABLE I. OPTIMIZED CP HUYGENS SOURCE ESA PARAMETERS (IN MM)

\begin{tabular}{|c|c|c|c|}
\hline$h 1=0.127$ & $h 2=0.508$ & $h 3=0.254$ & $h 4=0.127$ \\
\hline$L 1=1.48$ & $L 2=1$ & $L 3=0.16$ & $L 4=0.16$ \\
\hline$L 5=0.88$ & $L 6=0.7$ & $L 7=0.18$ & $W 1=1.48$ \\
\hline$W 2=0.13$ & $W 3=2.25$ & $W 4=1.14$ & $W 5=0.88$ \\
\hline$W 6=0.18$ & $W 7=0.7$ & $g 1=0.09$ & $g 2=0.09$ \\
\hline$R 1=1.5$ & $R 2=0.2$ & $R 3=0.6$ & $R 4=0.46$ \\
\hline$R 5=0.2$ & $R 6=0.15$ & $R 7=0.5$ & $R 8=0.6$ \\
\hline$\theta=100^{\circ}$ & \multicolumn{3}{|c|}{$N U L L$} \\
\hline
\end{tabular}

\section{PERFORMANCE OF HuYGENS SOURCE CP ESA}

As shown in Fig. 2, the HFSS (CST) simulated $-10-\mathrm{dB}$ impedance bandwidth is $400 \mathrm{MHz}$, from 27.93 to $28.33 \mathrm{GHz}$ (370 MHz, from 27.97 to $28.34 \mathrm{GHz}$ ) yielding FBW-10dB $\sim 1.41 \% \quad(\sim 1.31 \%)$. The predicted $3-\mathrm{dB}$ axial ratio (AR) bandwidth is $130 \mathrm{MHz}$, from 28.04 to $28.17 \mathrm{GHz}(120 \mathrm{MHz}$, from 28.07 to $28.19 \mathrm{GHz}$ ). The AR minimum value $1.18 \mathrm{~dB}$ $(1.86 \mathrm{~dB})$ occurs at $28.105 \mathrm{GHz}(28.14 \mathrm{GHz})$. The overall electrical size of the HFSS (CST) model at the lowest frequency bound of the AR bandwidth is $\mathrm{ka}=0.942$ (0.943). Within the 3-dB AR bandwidth, the FTBR values are higher than $20 \mathrm{~dB}(15 \mathrm{~dB})$ and their maximum value is $26.72 \mathrm{~dB}(17.9$ $\mathrm{dB})$ and is located at $28.11 \mathrm{GHz}(28.14 \mathrm{GHz})$. The peak realized gain varies from 1.80 to $2.03 \mathrm{dBi}$ (from 2.34 to 2.82 $\mathrm{dBi}$ ). The RE values are contained in the range from 69.6 to $73.4 \%$ (from 71.5 to $74.5 \%$ ).

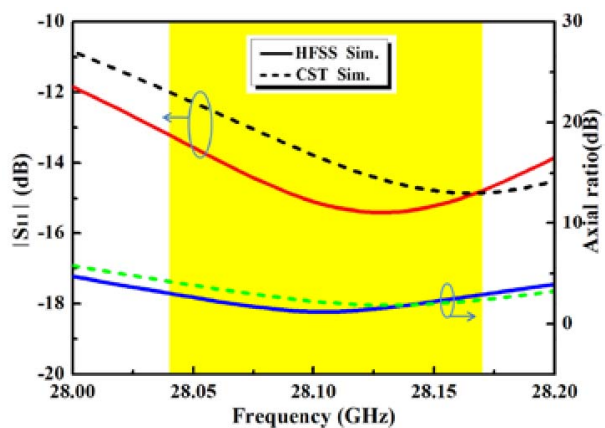

(a)

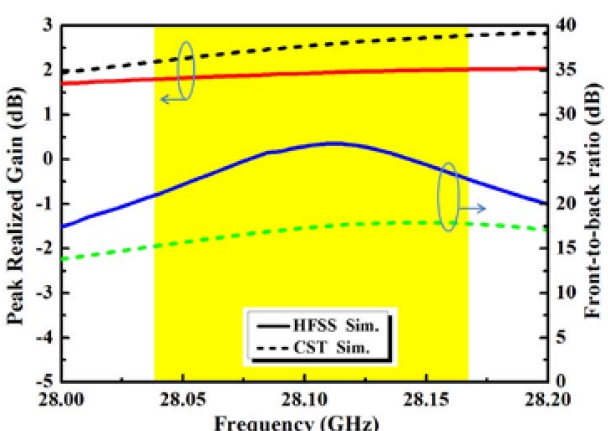

(b)

Fig. 2 The HFSS (solid line) and CST (dash line) simulated, and the measured (dash dot line) performance characteristics of the LP Huygens source ESA. (a) $\left|\mathrm{S}_{11}\right|$ values versus frequency, and (b) peak realized gain and and FTBR values versus frequency.

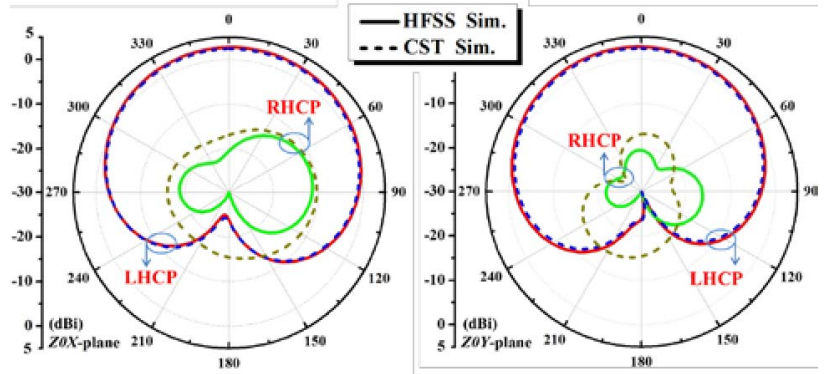

Fig. 3 The HFSS (solid line) and CST (dashed line) simulated 2D realized gain patterns of the optimized CP Huygens source ESA at its operational center frequency, $28.105 \mathrm{GHz}$, in the (a) zx and (b) yz planes. 
The realized gain radiation patterns of the $\mathrm{CP}$ Huygens source ESA at the $28.105 \mathrm{GHz}$ are shown in Fig. 3. The broadside realized gain is $1.94 \mathrm{dBi}(2.43 \mathrm{dBi})$ with the polarization purity higher than $20 \mathrm{~dB}(15 \mathrm{~dB})$, and the halfpower beamwidth coverage are from $-81^{\circ}$ to $99^{\circ}$ (from $-77^{\circ}$ to $90^{\circ}$ ) in E-plane and from $-93^{\circ}$ to $80^{\circ}$ (from $-84^{\circ}$ to $74^{\circ}$ ) in $\mathrm{H}-$ plane, respectively. It is easy to see that, in this design case, the arc strip has a clockwise rotation, therefore, the $\mathrm{CP}$ Huygens source ESA is LHCP. Vice versa, a RHCP Huygens source ESA could be designed by simple mirroring the feed lines on the Layer_4, simultaneously.

For health and safety purposes, the USA's Federal Communication Commission (FCC) has specified a $1.6 \mathrm{~W} / \mathrm{kg}$ limit of the SAR averaged over any $1.0 \mathrm{~g}$ of tissue in the shape of a cube (1-g average SAR) below $6 \mathrm{GHz}$ [9]. However, the permissible SAR levels at mmWave frequencies have not yet been officially released, even though the mmWave cellular system has become a hot research topic in recent year. In our studies, we used a Voxel Gustav human body in CST MWS to evaluate the SAR values produced by the CP Huygens source ESA.

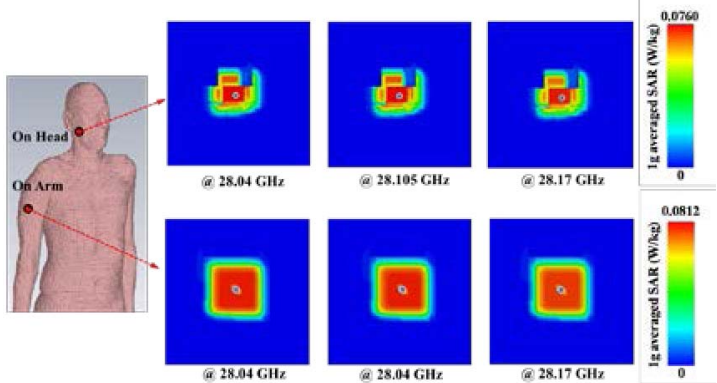

Fig. 4 Simulated $1 \mathrm{~g}$ averaged SAR values of the proposed Huygens source ESA mounted on the head and arm of the Voxel Gustav human body in CST MWS.

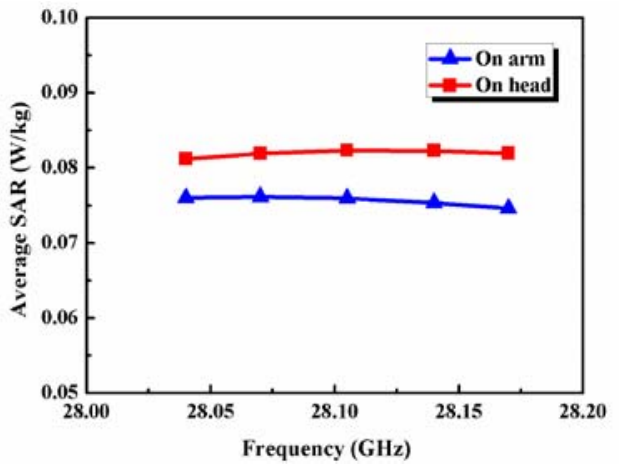

Fig. 5 Simulated peak 1g-averaged SAR values of the CP Huygens source ESA mounted on the head and the chest of the Voxel Gustav human body in CST MWS a function of the frequencies within its 3-dB AR operational bandwidth.

The Huygens source ESA is placed on the skin of the head and arm of the Voxel Gustav human body. The approximate placements and the relevant simulated $1 \mathrm{~g}$ averaged SAR values distributions are shown in Fig. 4. It is worth to mention that the skin tissue is not smooth in the model. It makes the simulated 1 g-averaged SAR values distributions in Fig. 4 not be gradual and smooth looking. In general, it could be observed that the SAR distributions in head and arm are both have the strongest values around the ESA and gradually weakened away from the ESA, at $28.04 \mathrm{GHz}, 28.105 \mathrm{GHz}$, and at $28.17 \mathrm{GHz}$, respectively. Furthermore, peak $1 \mathrm{~g}$ averaged SAR values of a series frequencies, from $28.04 \mathrm{GHz}$ to $28.17 \mathrm{GHz}$, is shown in Fig.5. Furthermore, the peak average SAR values on the head and on the arm for all of the frequencies tested are all below the maximum value: $0.085 \mathrm{~W} / \mathrm{kg}$, which is substantially lower than $0.15 \mathrm{~W} / \mathrm{kg}$ at $28 \mathrm{GHz}$ reported by Samsung Electronics [10].

\section{CONCLUSION}

A CP planar, multi-layer, broadside radiating, Huygens source ESAs based on multi-layer PCB technology operating in the mmWave $28 \mathrm{GHz}$ band was demonstrated. The simulated performance characteristics were obtained with two simulation environments, HFSS and CST MWS, and were shown to be in good agreement. The simulated SAR values were calculated with CST MWS. The Huygens source ESA should be very good candidates for the near-future $28 \mathrm{GHz}$ band, $5 \mathrm{G}$ systems.

\section{REFERENCES}

[1] T. Rappaport, et al, "Millimeter wave mobile communications for 5G cellular: It will work!," IEEE Access, vol. 1, pp. 335-349, 2013.

[2] P. Jin and R. W. Ziolkowski, "Metamaterial-inspired, electrically small Huygens sources," IEEE Antennas Wirel. Propag. Lett., vol. 9, pp. 501505, 2010.

[3] R. W. Ziolkowski, P. Jin, and C.-C. Lin, "Metamaterial-inspired engineering of antennas," Proc. IEEE, vol. 99, no. 10, pp. 1720-1731, Oct. 2011.

[4] P. Jin and R. W. Ziolkowski, "Metamaterial-inspired, electrically small Huygens sources," IEEE Antennas Wirel. Propag. Lett., vol. 9, pp. 501505, May, 2010.

[5] R. W. Ziolkowski, "Low profile, broadside radiating, electrically small Huygens source antennas," IEEE Access, vol. 3, pp. 2644-2651, Dec. 2015.

[6] M.-C. Tang, H. Wang, and R. W. Ziolkowski, "Design and testing of simple, electrically small, low-profile, Huygens source antennas with broadside radiation performance," IEEE Trans. Antennas Propag., vol. 64, no. 11, pp. 4607-4617, Nov. 2016.

[7] M.-C. Tang, B. Zhou, and R. W. Ziolkowski, "Low-profile, electrically small, huygens source antenna with pattern-reconfigurability that covers the entire azimuthal plane," IEEE Trans. Antennas Propag., vol. 65, no. 3, pp. 1063-1072, Mar. 2017.

[8] M.-C. Tang, T. Shi, and R. W. Ziolkowski, "Electrically small, broadside radiating Huygens source antenna augmented with internal non-Foster elements to increase its bandwidth," IEEE Antennas Wirel. Propag. Lett., vol. 16, Aug. 2017.

[9] IEEE standard for safety levels with respect to human exposure to radio frequency electromagnetic fields, $3 \mathrm{kHz}$ to $300 \mathrm{GHz}$, IEEE Standard C95.1-1999, 1999.

[10] W. Hong, K.-H. Baek, Y. Lee, Y. Kim, and S.-T. Ko, "Study and prototyping of practically large-scale mmWave antenna systems for $5 \mathrm{G}$ cellular devices," IEEE Communications Magazine, vol. 52, Iss. 9, pp. $63-69,2014$. 